\title{
Implementation of PHQ-9 Depression Screening for HIV-Infected Patients in a Real-World Setting
}

\author{
Malaika Edwards, MS [Depression Care Manager], \\ UNC Chapel Hill Infectious Diseases Clinic, Chapel Hill, NC, USA \\ Evelyn Quinlivan, MD [Associate Professor of Medicine] [Clinic Director], \\ UNC Chapel Hill Infectious Diseases Clinic, Chapel Hill, NC, USA \\ Kiana Bess, MA [Research Assistant], \\ Duke University Infectious Diseases Clinic, Durham, NC, USA \\ Bradley Gaynes, MD, MPH [Professor] [Director], \\ Psychiatric Outpatient Services, UNC Chapel Hill Department of Psychiatry, Chapel Hill, NC, USA
}

\section{Amy Heine, MSN, FNP [Instructor] [Program Director], \\ UNC Chapel Hill Infectious Diseases Clinic, Chapel Hill, NC, USA}

\author{
Anne Zinski, PhD [Assistant Professor of Medicine], \\ University of Alabama Birmingham 1917 Clinic, Birmingham, AL, USA \\ Riddhi Modi, MBBS, MPH [Research Assistant], and \\ University of Alabama Birmingham 1917 Clinic, Birmingham, AL, USA
}

Brian Pence, PhD, MPH [Associate Professor of Community and Family Medicine and Global Health]

Duke University, Durham, NC, USA

\begin{abstract}
The prevalence of depression is $20 \%-30 \%$ for people living with HIV, and while it is associated with poorer adherence to antiretrovirals, it is often unrecognized by medical providers. Although it has been challenging for some health care settings to develop consistent depression screening mechanisms, it is feasible to create screening protocols using the 9-item Patient Health Questionnaire (PHQ-9). Establishing a depression screening and response protocol is an iterative process that involves preparing staff, determining screening frequency, and developing procedures for response and appropriate medical record documentation. While there are multiple issues and potential challenges during implementation, it is possible to incorporate systematic depression
\end{abstract}

(C) 2013 Association of Nurses in AIDS Care. Published by Elsevier Inc. All rights reserved.

Publisher's Disclaimer: This is a PDF file of an unedited manuscript that has been accepted for publication. As a service to our customers we are providing this early version of the manuscript. The manuscript will undergo copyediting, typesetting, and review of the resulting proof before it is published in its final citable form. Please note that during the production process errors may be discovered which could affect the content, and all legal disclaimers that apply to the journal pertain.

Disclosure statement: The authors report no real or perceived vested interests related to this article that could be construed as a conflict of interest. 
screening into HIV primary care in a manner that achieves staff buy-in, minimizes patient burden, streamlines communication, and efficiently uses the resources available in the medical setting.

\section{Keywords}

depression; HIV; infectious diseases; PHQ-9; screening

Depression is a common co-morbidity in HIV-infected patient populations, with prevalence rates of 20\%-30\% (Bing et al., 2001; Pence, O'Donnell, \& Gaynes, 2012), but it is often unrecognized, undiagnosed, and undertreated by medical providers (Asch et al., 2003; Gilbody, Sheldon, \& Wessely, 2006). The co-occurrence of depression in HIV-infected patients is associated with poorer adherence to antiretrovirals and other negative clinical outcomes (Crane et al., 2010; Leserman et al., 2007; Pence, Miller, Gaynes, \& Eron, 2007), but early identification and treatment of depression has the potential to impact HIV treatment outcomes (Tegger et al., 2008; Tsai et al., 2010).

In 2000, in order to screen for mental illness and substance abuse, the Infectious Diseases (ID) clinics at Duke University Medical Center and the University of North Carolina-Chapel Hill Health Care System (UNC) used the Substance Abuse and Mental Illness Symptoms Screener (SAMISS), a simple tool to identify likely cases in HIV-infected patient populations. Clinic-wide screening confirmed that mental illness was highly prevalent (Pence, Miller, Whetten, Eron, \& Gaynes, 2006; Whetten et al., 2005) and the SAMISS was shown to have high sensitivity, detecting $95 \%$ of patients with confirmed mental illness (Pence et al., 2005). Depressive disorders were the most frequently identified mental illness, but specificity was only moderate, and $50 \%$ of the patients with a positive screen result for symptoms of mental illness did not meet clinical criteria for a mood or anxiety disorder. A tool to distinguish clinical depression from clinically non-significant depressive symptoms was lacking, and a feasible, accurate procedure for screening was needed. Such a procedure could provide better identification and, ultimately, management of depressive illness in HIV-infected patients.

While evidence exists to support the importance of depression screening (O'Connor, Whitlock, Beil, \& Gaynes, 2009) and best practice guidelines recommend routine assessments using standardized measures (American Psychiatric Association, 2010), barriers have been identified that contribute to low depression screening rates within health systems (Harrison, Miller, Schmitt, \& Touchet, 2010), and medical environments have found it difficult to develop consistent depression screening mechanisms (Shacham, Nurutdinova, Satyanarayana, Stamm, \& Overton, 2009). To address the need for an efficient screening tool and a systematic process, and to improve the identification of patients with depressive symptoms, the Duke and UNC ID clinics successfully implemented screening protocols using a carbon copy paper version of the 9-item Patient Health Questionnaire (PHQ-9). Drawing on these experiences, this paper describes the process and reports key considerations for successful implementation of routine depression screening in busy, academic infectious diseases clinics. 


\section{Setting}

The ID clinics at Duke, a private hospital, and UNC, a public hospital, are large academic clinics with patient populations greater than 1,700 that have provided HIV care in central North Carolina for more than 30 years. The demographics of the two patient populations are very similar (Table 1). Reflective of HIV in the southeastern United States, $28 \%$ of the population is female, a small but growing minority is Latino/a (4\%), and African Americans are the overwhelming majority population $(57 \%)$.

\section{PHQ-9 Screening Evolution}

\section{Overview}

Both the Duke and UNC sites developed a process for routine, real-time depression screening in conjunction with integrating new depression treatment services as part of the SLAM DUNC Study (R01MH086362: Strategies to Link Antiretroviral and Antidepressant Management at Duke, UAB and UNC). The SLAM DUNC Study is a randomized clinical trial to evaluate the effect of depression management on antiretroviral medication adherence (Pence, Gaynes, et al., 2012). In the study, Depression Care Managers (DCMs) worked with HIV care providers to optimize antidepressant therapy for HIV-infected patients with depression. The present paper focuses on lessons learned from developing screening protocols at the Duke and UNC study sites; the University of Alabama at Birmingham (UAB) study site utilized a depression screening and response protocol that was in place prior to study launch, which has been described previously (Lawrence et al., 2010).

The development of a robust depression screening and response protocol at these two sites was an iterative process that required consideration of multiple issues. These issues included preparing staff and HIV providers for the new protocol; selecting the frequency at which to screen patients; managing distribution, retrieval, and review of screeners; responding to positive depression screens and endorsements of suicidal ideation (SI); and establishing electronic medical record (EMR) documentation procedures.

\section{Preparing Staff and Providers}

In order to implement a successful screening procedure, it is crucial to acknowledge that all clinic personnel may have a role in the process, whether through distribution of the questionnaire or through patient follow-up. This involvement includes front desk and nursing staff, research personnel, behavioral health professionals, and HIV care providers.

Both the Duke and UNC clinics encouraged feedback from HIV care providers through meetings, personal communication, or via email to increase support for the new process and to aid in developing buy-in. Before initiating screening, the UNC site also held meetings with all clinic personnel to solicit staff support and garner suggestions for process implementation, so as to not significantly increase burden or create inefficiency for anyone in the clinic. 


\section{Selecting the Screening Frequency}

Prior to screening implementation, all patients needed to be assessed; therefore, the Duke and UNC clinics initiated universal screening of all scheduled HIV-infected patients. After approximately 7 months of universal screening, "screener fatigue" among patients with frequent appointments became evident at both sites. Due to screener fatigue, and because an optimal depression screening frequency has not yet been defined (Harrison et al., 2010), each site then implemented a manual tracking system that provided a prompt for screening at 6-month intervals. The resulting reduction in volume also lowered the number of lost screeners and allowed staff more time to assist patients who expressed reluctance to complete the screener due to low literacy. Alternate flagging mechanisms in the electronic medical record (EMR) or other scheduling systems may also be used, as determined by the available resources in a given setting.

\section{Managing the Distribution of Screeners}

In an effort to effectively manage the flow of paperwork, each site developed a procedure for administering PHQ-9s to patients who were due for screening at each 6-month interval. Both sites used a staff member, in this case the DCM, to pre-screen the clinic schedule and manually document the most recent screening date and results. However, the procedures for selecting patients for screening, retrieving screeners, and reviewing results varied according to the unique staff composition and culture at each location.

At the Duke site, the DCM (or study staff member) identified patients due for screenings and attached the PHQ-9 to designated patients' encounter forms on the day before their provider visits (Figure 1A). Employing study staff to maintain primary responsibility for administering the questionnaire considerably reduced the burden on other clinic staff.

Conversely, incorporating the front desk in the distribution process proved helpful at UNC. UNC's DCM reviewed and annotated a paper version of the clinic schedule 24 hours in advance to flag patients who should be screened. The front desk staff received a copy of the annotated schedule and used it to distribute the PHQ-9 (Figure 2A).

\section{Retrieving Results of Screening}

Once patients received PHQ-9s, HIV care providers had to review the results before or during the clinic visit to address screening results in real-time. However, clinic staff also needed access to screening results in order to assist with tracking, responding to positive results, and completing EMR documentation. To achieve this end, each site determined that patients should return completed PHQ-9s to the front desk. The DCM reviewed the results and took positive screens of 10 or more directly to the medical provider or appropriate behavioral staff member (Figures 1A and 2A).

This system produced a high rate of questionnaire return from patients but relied on staff to retrieve and score screeners, then deliver results to providers in the short time between patient triage and medical exam. It was difficult for HIV providers to review the screener and discuss the findings with patients in real-time with this approach. 


\section{Reviewing Results of Screening}

To deliver screening results to providers in real-time and better integrate the screening results into the medical encounter at the Duke site, the triage nurse began to assist with collection of PHQ-9s and attached them to providers' paperwork for review during the appointment. To increase the utility of the screening for providers, the PHQ-9 was combined with an additional set of brief behavioral health screening questions addressing other symptoms of psychological distress, alcohol and drug use, antiretroviral adherence, and smoking, to create the comprehensive Duke ID Clinic Behavioral Screener, as well as a onepage general review of physical systems. Patients were then instructed to give the Behavioral Screener to their providers and, following the appointment, providers were asked to leave the screener in a secure central location where the DCMs collected the form and addressed the results as appropriate. While this process allowed providers to incorporate the PHQ-9 results into the medical appointment in real time and increased their investment in the screening process, the return rate of the Behavioral Screener to the DCM was low and the behavioral health staff members were unable to consistently provide assistance for tracking, follow-up, and documentation.

Similar to the Duke site, UNC adapted their procedures to better integrate the screener into clinic operations and ensure that providers received the completed PHQ-9 before the medical exam. As a result, the nurses at UNC were charged with collecting PHQ-9 screeners from patients at triage. Once collected, the nurse performed an interim step of reviewing the suicidality question for any endorsement of suicidal ideation (SI). Screeners with a positive endorsement of SI were immediately routed to the DCM, behavioral health clinician, or HIV care provider to ensure that patients who reported SI received a safety assessment before leaving the clinic. If there was no endorsement of SI, the nurse placed the questionnaire in a designated secure location for collection by the DCM or other study staff. This system produced further challenges because the burden of finding staff to respond to positive screeners was transferred to the nurses during triage.

The DCM at UNC continued to be responsible for discussing all scores above 10, with or without SI, with the appropriate provider at the time of the patient's visit. Although UNC's DCM made efforts to discuss screening results before the provider saw the patient in the exam room, this was a difficult task, particularly during high-volume clinic sessions.

\section{Reducing Screener Loss Rates}

To reduce screener loss rates and improve communication of results to both providers and DCMs, the Duke site, using their combined Behavioral Screener, and UNC, using the PHQ-9, transitioned to carbon copy paper. The designated staff member at each site routed one copy to the provider and the other copy to the DCM. The carbon copy screener helped providers immediately respond to screening results and still allowed the DCM or behavioral health team member access to the results for tracking, assistance with follow-up, and documentation. 


\section{Summarizing the Current Screening Procedures}

When the Duke and UNC clinics finalized their procedures for implementing depression screening after the first year, one staff member, at these sites the DCM, pre-screened patients with upcoming appointments and identified those due for their 6-month screening. Using carbon copy paper screeners, the front desk staff distributed the forms to patients along with other clinic paperwork (Figures 1B and 2B).

Because of the Duke ID clinic structure, it was optimal to instruct patients to return screeners to the triage nurse, who was then responsible for delivery to the provider or another staff member for review, assistance with addressing reports of depression and SI, and the resulting documentation (Figure 1B). Staff at the UNC ID clinic found that asking patients to return screeners to the front desk helped streamline the process. Front desk staff separated carbon copies and attached one copy to the providers' paperwork and a designated clinic staff member periodically checked the front desk for the other copy of the screener (Figure 2B). As each process demonstrates, the sites resolved the issue of permitting the medical staff and the behavioral health team to review screening results and act promptly by migrating to a carbon copy form, although information technology solutions could also be used depending on available resources.

\section{Responding to Endorsements of Depression and Suicidal Ideation}

Although the threshold for staff response at Duke was set at greater than or equal to 10 from the start of the implementation process, at UNC, the social work staff was initially dispatched to respond to every PHQ-9 score greater than or equal to 5 (the threshold for mild depressive symptoms). This proved to be challenging due to the high volume of scores in this range and responders recognized that scores classified as mild (5-9) were more frequently due to chronic somatic complaints rather than primary mood disorders. As a result, the staff response threshold was changed to a PHQ-9 score greater than or equal to 10 or any report of suicidality, either of which would require an intervention by provider, DCM, or social work staff.

DCMs responding to positive depression screeners followed up by offering participation in the depression study. In addition, depending on the patient's needs, resources, and location, the DCM referred patients to clinic social workers, county mental health agencies for assessment and treatment, hospital psychiatric services, addictions services, or domestic violence programs. For patients with difficulty accessing mental health services, the depression study at Duke and UNC offered a unique opportunity to gain entry into care that was not immediately available.

At program launch, all PHQ-9s with any endorsement of suicidality (i.e., a response to question 9 of a few days, more than half the days, or nearly every day) were flagged for a response by the behavioral health team, regardless of the PHQ-9 total score. However, most cases were determined to be chronic, passive ideation of which the providers were already aware. Routing PHQ-9s directly to the social work team to address SI reports proved to be an unnecessary duplication of effort, as the provider typically had more familiarity with the patient and had already conducted a suicide assessment. Ultimately, staff determined that 
any SI endorsement should be brought to the immediate attention of the HIV care provider, who would then decide whether to follow up directly, refer to the social work team, DCM, or to psychiatry for additional assessment or intervention.

At both sites, when patients reported suicidality, clinic staff or providers conducted a comprehensive, structured suicide risk assessment. Most reports of suicidality were passive, and required no response beyond medical provider follow-up in the course of administering HIV care. If the patient endorsed active ideation without intent, means, or a plan for selfharm, the responding staff member and patient developed a safety plan. Members of the behavioral health team escorted patients to the Emergency Department for psychiatric stabilization when the patient could not develop a safety plan and when the patient was determined to be an imminent danger to him or herself.

\section{Documentation}

Initially, the DCMs entered PHQ-9 scores into patients' medical records. All scores, with special attention to those greater than or equal to 10 or any endorsement of SI, were documented. At the Duke site, the DCM transitioned to documenting only PHQ-9 scores greater than or equal to 10 in the medical record in order to reduce the work burden. UNC continued to document all scores in the medical record for the tracking of outcomes. As providers became accustomed to using the tool, they also included patient scores and outcomes in their medical record notes.

\section{Discussion}

As ID clinics consider implementing a depression screening process, such as using the PHQ-9, at least three major areas of tension will need consideration. The first is whether the screening results will be evaluated in real-time versus a delayed evaluation. Both the Duke and UNC ID clinics attempted multiple process iterations to ensure that screeners would be reviewed in real time. Each site focused on addressing reports of depression during inperson interactions to maintain appropriate standards of care and to allow patients to benefit from immediate communication with staff about the need for intervention.

The next consideration was whether the screener should go directly to the HIV care provider, the behavioral health provider, or both. After trying a range of approaches, both sites finally decided that ID providers and DCMs should each receive the PHQ-9 through the use of a carbon copy version. This made certain that multiple clinic staff members were aware of patient reports and could provide a suitable intervention as soon as possible.

The final issue to address was how to develop a process for attending to scores greater than or equal to 10 and SI. In order to provide effective real-time responses to positive screens, it proved best in both clinics to have the DCM and the medical provider alerted simultaneously through the use of carbon copy screeners. Ultimately, the clinics' protocols for responding to SI helped determine the appropriateness of real-time over delayed followup. Patients who endorsed suicidality needed to be brought to the attention of the provider in order to maintain appropriate standards of medical care and ensure that the patient's needs 
were met as soon as possible. Real-time follow-up also gave the provider the primary review of the PHQ-9.

\section{Successes}

Perhaps the most important accomplishment of this endeavor was the change to care delivery with the adoption of a standardized depression screening instrument administered at 6-month intervals as part of routine HIV care, combined with appropriate evaluation and response. Not only did the introduction of this process give providers a tool for reviewing depressive symptoms, it highlighted the need for a standardized risk assessment to help distinguish between passive and active SI. As such, HIV care providers requested additional training to assess SI, and an algorithm to rate type, severity, and acuity of SI. An additional success, resulting from the coordinated effort to introduce the screening process in the HIV clinics, was that across clinic positions, staff tried to ensure that patients who reported distress were assisted before leaving the clinic.

\section{Challenges}

One of several challenges to emerge when these sites implemented depression screening was the risk of losing screeners with high scores or endorsements of SI. Therefore, staff developed a carbon copy of the PHQ-9 to minimize burden on clinic staff while ensuring the execution of an appropriate response to such reports.

The ID clinics also needed to determine the best way to distribute screeners to HIV-infected patients while maintaining standards of confidentiality. Neither clinic used indicators on the Behavioral Screener or PHQ-9 to specify that the assessments were only for HIV-infected patients. At the Duke clinic, the front desk staff placed the screener on a clipboard and distributed it to patients at check-in. The UNC clinic staff addressed confidentiality by placing the PHQ-9 in manila folders before distributing to patients.

Limited access to mental health care for many patients became a key issue when depressive symptoms were identified and a plan for intervention was required. Even when referrals were made to mental health agencies, it was often several months before patients were able to secure an appointment. As part of the implementation process, developing intermediate solutions to address access to care issues was necessary. In settings where a depression study is not available as an intervention, it may be feasible to obtain a psychiatric consultation; refer to clinical social workers or other behavior health professionals to provide counseling; or consider whether the HIV care provider could take on the role of treating depression, as many primary care providers do now, while waiting for the patient to become established in psychiatric or other mental health care.

Patients with limited literacy also posed unique challenges. Due to stigma, patients may opt not to disclose low literacy and may instead refuse to complete the screener. It was important to train staff to offer to read the questions in a manner that was sensitive to the stigma of illiteracy for those who expressed reluctance to complete the screener or those who were identified as having literacy barriers. 


\section{Conclusion}

It is feasible to use the PHQ-9 as a clinic-wide screening tool to identify depressive symptoms in HIV-infected patients receiving HIV primary care in academic medical clinics. An effective approach to introducing a depression screening protocol should minimize burden on patients and clinic staff, in addition to achieving staff buy-in by collectively discussing roles and how screening might complement existing responsibilities. Staff should also consider the type and frequency of screening to avoid screening fatigue and allow clinic staff more time to identify and address patients with true depression in real time. Screening type and frequency will likely be based on the resources available in unique medical settings. Furthermore, developing appropriate responses to depressive categories and reports of SI or patients in crisis is critical. Over time, screening at 6-month intervals has continued to help monitor patient symptoms, with minimal screening fatigue, and has decreased the follow-up burden on clinic and study staff. Finally, streamlined communication between staff and HIV care providers is also necessary, and should take into account specific protocols for routing screeners and completing documentation.

The experiences at these two ID clinics highlighted central issues that would be important to consider in any clinic considering depression screening. They also demonstrate that, with flexibility and solution-oriented thinking, these challenges can be successfully navigated and routine depression screening can be effectively integrated into routine care in a resourceefficient manner.

\section{References}

American Psychiatric Association. Practice guideline for the treatment of patients with major depressive disorder. 3rd ed.. Author; Arlington, VA: 2010.

Asch SM, Kilbourne AM, Gifford AL, Burnam MA, Turner B, Shapiro MF, Bozzette SA. Underdiagnosis of depression in HIV: Who are we missing? Journal of General Internal Medicine. 2003; 18(6):450-460. doi:10.1046/j.1525-1497.2003.20938.x. [PubMed: 12823652]

Bing EG, Burnam A, Longshore D, Fleishman JA, Sherbourne CD, London AS, Shapiro M. Psychiatric disorders and drug use among human immunodeficiency virus-infected adults in the United States. Archives of General Psychiatry. 2001; 58(8):721-728. doi:10.1001/archpsyc. 58.8.721. [PubMed: 11483137]

Crane PK, Gibbons LE, Willig JH, Mugavero MJ, Lawrence ST, Schumacher JE, Crane HM. Measuring depression levels in HIV-infected patients as part of routine clinical care using the nineitem Patient Health Questionnaire (PHQ-9). AIDS Care. 2010; 22(7):874-885. doi: 10.1080/09540120903483034. [PubMed: 20635252]

Gilbody S, Sheldon T, Wessely S. Health policy: Should we screen for depression? British Medical Journal. 2006; 332(7548):1027-1030. doi:10.1136/bmj.332.7548.1027. [PubMed: 16644833]

Harrison DL, Miller MJ, Schmitt MR, Touchet BK. Variations in the probability of depression screening at community-based physician practice visits. Primary Care Companion to the Journal of Clinical Psychiatry. 2010; 12(5):e1-e8. doi:10.4088/PCC.09m00911blu.

Lawrence ST, Willig JH, Crane HM, Ye JT, Aban I, Lober W, Schumacher JE. Routine, selfadministered, touch-screen, computer-based suicidal ideation assessment linked to automated response team notification in an HIV primary care setting. Clinical Infectious Diseases. 2010; 50(8): 1165-1173. doi:10.1086/651420. [PubMed: 20210646]

Leserman J, Pence BW, Whetten K, Mugavero MJ, Thielman NM, Swartz MS, Stangl D. Relation of lifetime trauma and depressive symptoms to mortality in HIV. American Journal of Psychiatry. 2007; 164(11):1707-1713. doi:10.1176/appi.ajp.2007.06111775. [PubMed: 17974936] 
O'Connor EA, Whitlock EP, Beil TL, Gaynes BN. Screening for depression in adult patients in primary care settings: A systematic evidence review. Annals of Internal Medicine. 2009; 151(11): 793-803. doi:10.7326/0003-4819-151-11-200912010-00007. [PubMed: 19949145]

Pence BW, Gaynes BN, Whetten K, Eron JJ Jr. Ryder RW, Miller WC. Validation of a brief screening instrument for substance abuse and mental illness in HIV-positive patients. Journal of Acquired Immune Deficiency Syndromes. 2005; 40(4):434-444. [PubMed: 16280698]

Pence BW, Gaynes BN, Williams Q, Modi R, Adams J, Quinlivan EB, Mugavero MJ. Assessing the effect of measurement-based care depression treatment on HIV medication adherence and health outcomes: Rationale and design of the SLAM DUNC Study. Contemporary Clinical Trials. 2012; 33(4):828-838. doi:10.1016/j.cct.2012.04.002. [PubMed: 22542960]

Pence BW, Miller WC, Gaynes BN, Eron JJ Jr. Psychiatric illness and virologic response in patients initiating highly active antiretroviral therapy. Journal of Acquired Immune Deficiency Syndromes. 2007; 44(2):159-166. doi:10.1097/QAI.0b013e31802c2f51. [PubMed: 17146374]

Pence BW, Miller WC, Whetten K, Eron JJ, Gaynes BN. Prevalence of DSM-IV-defined mood, anxiety, and substance use disorders in an HIV clinic in the Southeastern United States. Journal of Acquired Immune Deficiency Syndromes. 2006; 42(3):298-306. doi:10.1097/01.qai. 0000219773.82055.aa. [PubMed: 16639343]

Pence BW, O'Donnell JK, Gaynes BN. Falling through the cracks: The gaps between depression prevalence, diagnosis, treatment, and response in HIV care. AIDS. 2012; 26(5):656-658. doi: 10.1097/QAD.0b013e3283519aae. [PubMed: 22398574]

Shacham E, Nurutdinova D, Satyanarayana V, Stamm K, Overton ET. Routine screening for depression: Identifying a challenge for successful HIV care. AIDS Patient Care and STDs. 2009; 23(11):949-955. doi:10.1089/apc.2009.0064. [PubMed: 19925308]

Tegger MK, Crane HM, Tapia KA, Uldall KK, Holte SE, Kitahata MM. The effect of mental illness, substance use, and treatment for depression on the initiation of highly active antiretroviral therapy among HIV-infected individuals. AIDS Patient Care STDs. 2008; 22(3):233-243. doi:10.1089/ apc.2007.0092. [PubMed: 18290749]

Tsai AC, Weiser SD, Petersen ML, Ragland K, Kushel MB, Bangsberg DR. A marginal structural model to estimate the causal effect of antidepressant medication treatment on viral suppression among homeless and marginally housed persons With HIV. Archives of General Psychiatry. 2010; 67(12):1282-1290. doi:10.1001/archgenpsychiatry.2010.160. [PubMed: 21135328]

Whetten K, Reif SS, Napravnik S, Swartz MS, Thielman NM, Eron JJ, Soto T. Substance abuse and symptoms of mental illness among HIV-positive persons in the southeast. Southern Medical Journal. 2005; 98(1):9-14. doi:10.1097/01.Smj.0000149371.37294.66. [PubMed: 15678633] 


\section{Key Considerations}

- Systematic depression screening can improve the identification and management of depressive illness in HIV-infected patients.

- Developing a protocol for routine depression screening in HIV primary care is an iterative process requiring multiple considerations involving staff, patients, and providers.

- Successful screening implementation achieves staff buy-in, minimizes patient burden, determines screening intervals, streamlines communication, outlines responses to endorsements of depression and crisis situations, and ensures appropriate medical record documentation. 
A. First screening process iteration

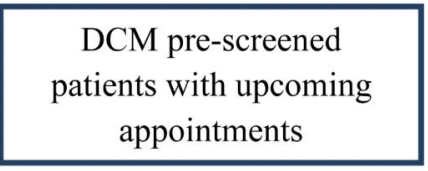

DCM flagged all patients eligible for screening at each visit
Designated staff reviewed

PHQ-9s and gave results to providers

Front desk staff kept PHQ-9s at the front.

Front desk distributed

PHQ-9s to designated patients

Patients returned completed PHQ-9s to the front desk

B. Final screening process

DCM pre-screened patients with upcoming appointments

DCM flagged all patients eligible for screening at 6month intervals

Front desk distributed carbon copy Behavioral Screener to designated patients
Designated staff reviewed Behavioral Screener carbon copies to facilitate consultation

Patients returned completed

Behavioral Screeners to triage

Nurse delivered one copy

to provider and one copy

to DCM

nurse

Figure 1.

Evolution of screening process at Duke Infectious Diseases.

Note. DCM = Depression Care Manager; PHQ-9 = 9-item Patient Health Questionnaire;

Bold, italicized text $=$ a screening process modification. Bold textbox $=$ the introduction of $\mathrm{a}$ new step. 
A. First screening process iteration

DCM pre-screened patients with upcoming appointments
Designated staff reviewed PHQ-

9s and gave results to providers

Front desk staff kept

PHQ-9s at the front. patients eligible for screening at each visit

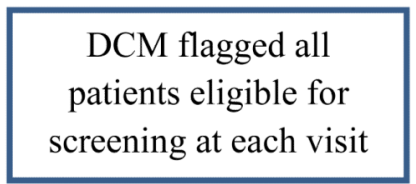
Front desk distributed
PHQ-9s to designated patients

Patients returned completed PHQ-9s to the front desk

B. Final screening process

DCM pre-screened patients

with upcoming appointments

DCM flagged all patients

eligible for screening at 6month intervals

Front desk distributed carbon copy PHQ-9 to designated patients
Designated staff reviewed PHQ-9 carbon copies to facilitate consultation
Provider had access to PHQ-

9 during exam

Patients returned completed

PHQ-9s to the front desk

Front desk staff attached one copy to provider paperwork and left the other at the front

Figure 2.

Evolution of screening process at University of North Carolina Infectious Diseases.

Note. DCM = Depression Care Manager; PHQ-9 = 9-item Patient Health Questionnaire;

Bold, italicized text $=$ a screening process modification. Bold textbox $=$ the introduction of $a$ new step. 
Table 1

Demographic Characteristics of the Implementation Site Populations

\begin{tabular}{|l|l|r|r|r|r|}
\hline & & \multicolumn{2}{|c|}{ UNC-CH } & \multicolumn{2}{c|}{ DUKE } \\
\hline Demographic & \multicolumn{1}{|c|}{ Category } & \multicolumn{1}{c|}{$\boldsymbol{N}$} & \multicolumn{1}{c|}{$\%$} & \multicolumn{1}{c|}{$\boldsymbol{N}$} & \multicolumn{1}{c|}{$\%$} \\
\hline Population & Total & 1711 & $100 \%$ & 1794 & $100 \%$ \\
\hline Gender & Women & 493 & $29 \%$ & 504 & $28 \%$ \\
\hline & Men & 1212 & $71 \%$ & 1290 & $72 \%$ \\
\hline & Transgender & 6 & $<1 \%$ & unknown & \\
\hline Age & $13-24$ & 98 & $6 \%$ & 51 & $3 \%$ \\
\hline & $25-44$ & 688 & $40 \%$ & 669 & $37 \%$ \\
\hline & $45-64$ & 864 & $50 \%$ & 998 & $56 \%$ \\
\hline & 265 & 61 & $4 \%$ & 76 & $4 \%$ \\
\hline Race/Ethnicity & White, non-Hispanic & 517 & $30 \%$ & 625 & $35 \%$ \\
\hline & Black, non-Hispanic & 972 & $57 \%$ & 1033 & $57 \%$ \\
\hline & Hispanic, all races & 116 & $7 \%$ & 66 & $4 \%$ \\
\hline & Other, non-Hispanic & 106 & $6 \%$ & 70 & $4 \%$ \\
\hline HIV RNA & $<400$ & 1403 & $82 \%$ & 1487 & $83 \%$ \\
\hline & $\geq 400$ & 308 & $18 \%$ & 297 & $16 \%$ \\
\hline
\end{tabular}

Note. $\mathrm{RNA}=$ ribonucleic acid 